\title{
Giant Dipole Resonances in Odd-Mass Nuclei and the Interacting Boson-Fermion Model.
}
A. Ventura $\left({ }^{1}\right)$, G. Maino $\left(^{1}\right)$ and L. Zuffi $\left({ }^{2}\right)$
(1) Ente Nuove Tecnologie, Energia e Ambiente - Bologna INFN, Sezione di Firenze - Firenze
(2) Dipartimento di Fisica dell'Università - Milano INFN, Sezione di Milano - Milano

(Nuovo Cimento A, 107, 43, (1994))

PACS 21.60.Fw - Models based on group theory.

PACS 24.30.Cz - Giant resonances.

PACS 25.20.Dc - Photon absorption and scattering.

PACS 99.10 - Errata.

Due to an unpleasant mistake, fig. 3 and 5 were exchanged after the proofreading. We apologize to the authors for the inconvenience. The corrected figures are shown below.
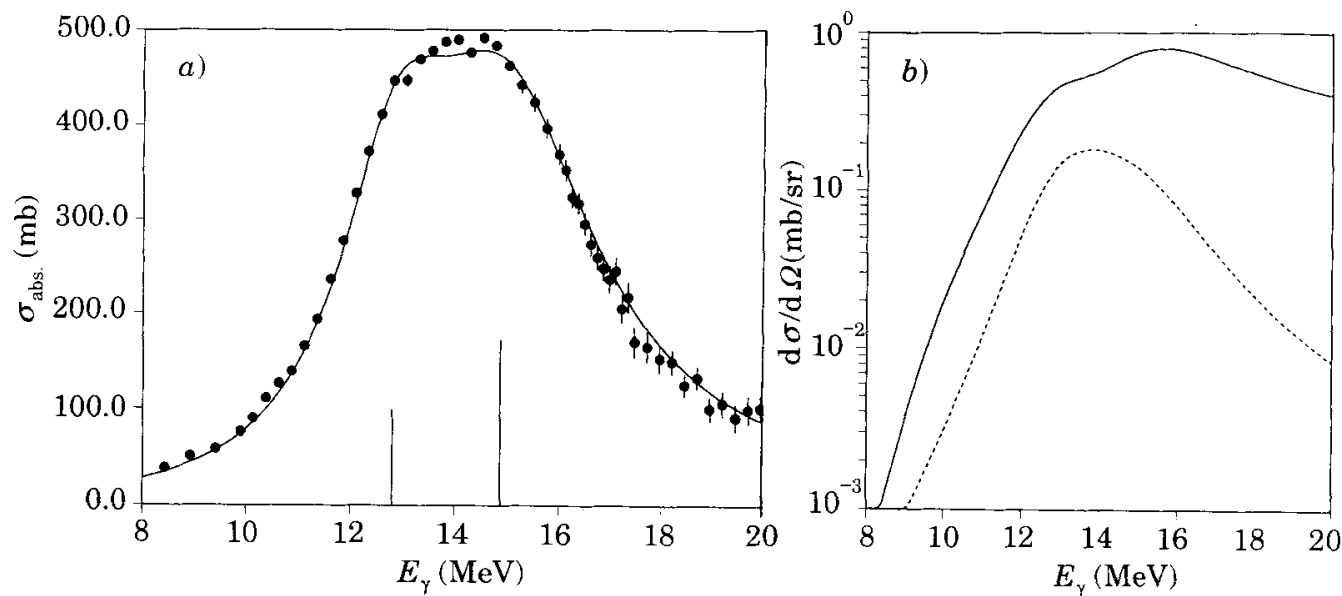

Fig. 3. - Photon absorption and scattering by ${ }^{188}$ Os. a) Photoabsorption cross-section, with the experimental data of ref.[18]. The vertical bars at the GDR energies are $B(E 1)$ strengths in arbitrary units. $b$ ) Solid curve: elastic scattering; dashed curve: inelastic scattering to the $2_{1}^{+}$ state $\theta=140^{\circ}$. 

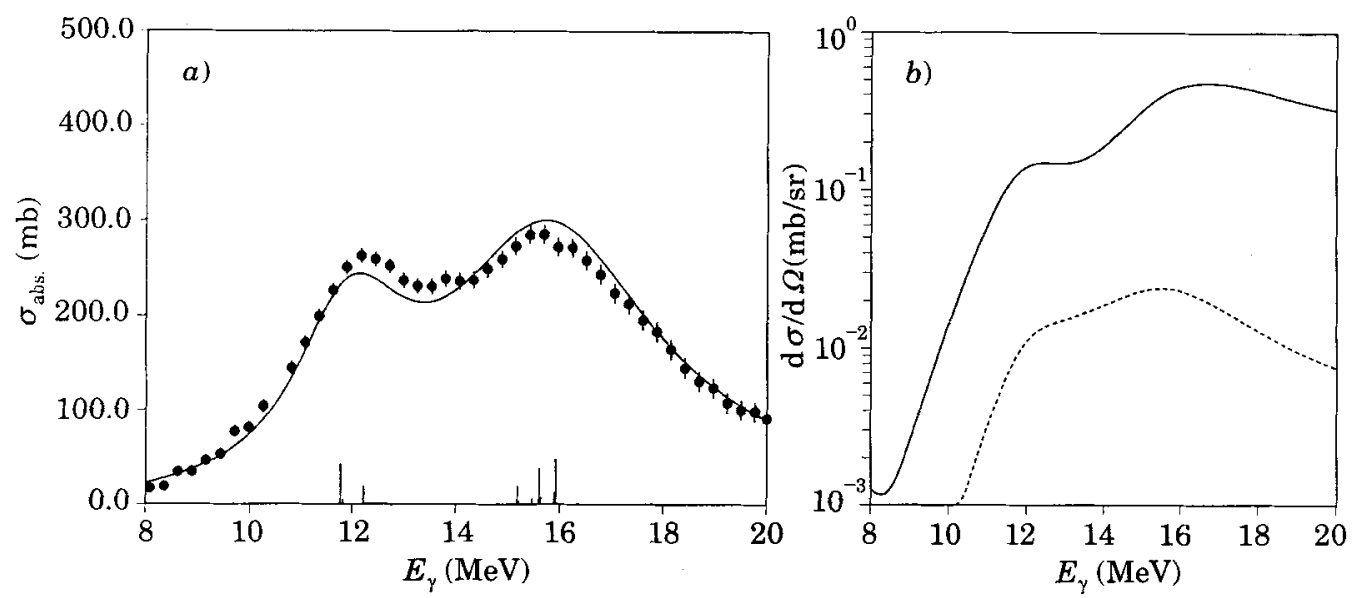

Fig. 5. - Photon absorption and scattering by ${ }^{159} \mathrm{~Tb} . a$ ) Calculated photoabsorption cross-section; the experimental data are taken from ref. [24]. The vertical bars are $B(E 1)$ strengths in arbitrary units. b) Solid line: elastic scattering; dashed line: cumulative inelastic scattering to the $(5 / 2)_{1}^{+}$ and $(7 / 2)_{i}^{-}$states, $\theta=140^{\circ}$. 